\title{
CONVOLUTION IDENTITIES FOR CAUCHY NUMBERS OF THE SECOND KIND
}

\author{
Takao KOMATSU
}

(Received 17 March 2014 and revised 30 June 2014)

\begin{abstract}
Let $\hat{c}_{n}$ be the $n$th Cauchy number of the second kind, defined by the generating function $x /(1+x) \ln (1+x)=\sum_{n=0}^{\infty} \hat{c}_{n} x^{n} / n$ !. We obtain explicit expressions for $\left(\hat{c}_{l}+\right.$ $\left.\hat{c}_{m}\right)^{n}:=\sum_{j=0}^{n}\left(\begin{array}{l}n \\ j\end{array}\right) \hat{c}_{l+j} \hat{c}_{m+n-j}$ with arbitrary fixed integers $l, m \geq 0$. The corresponding expression for Bernoulli numbers has been studied by Agoh and Dilcher, and that for Cauchy numbers of the first kind by the author.
\end{abstract}

\section{Introduction}

The Cauchy numbers of the second kind $\hat{c}_{n}(n \geq 0)$ are defined by

$$
\hat{c}_{n}=\int_{0}^{1}(-x)(-x-1) \cdots(-x-n+1) d x
$$

and the generating function of $\hat{c}_{n}$ is given by

$$
\frac{x}{(1+x) \ln (1+x)}=\sum_{n=0}^{\infty} \hat{c}_{n} \frac{x^{n}}{n !} \quad(|x|<1)
$$

(see $[4,10])$. Several initial values are

$$
\begin{gathered}
\hat{c}_{0}=1, \quad \hat{c}_{1}=-\frac{1}{2}, \quad \hat{c}_{2}=\frac{5}{6}, \quad \hat{c}_{3}=-\frac{9}{4}, \quad \hat{c}_{4}=\frac{251}{30}, \\
\hat{c}_{5}=-\frac{475}{12}, \quad \hat{c}_{6}=\frac{19087}{84}, \quad \hat{c}_{7}=-\frac{36799}{24} .
\end{gathered}
$$

With the classical umbral calculus notation (see, e.g., [11]), define $\left(\hat{c}_{l}+\hat{c}_{m}\right)^{n}$ for $l, m, n \geq$ 0 by

$$
\left(\hat{c}_{l}+\hat{c}_{m}\right)^{n}:=\sum_{j=0}^{n}\left(\begin{array}{l}
n \\
j
\end{array}\right) \hat{c}_{l+j} \hat{c}_{m+n-j} .
$$

The similar concepts about the Bernoulli numbers $B_{n}$ and the Cauchy numbers of the first kind have been studied by extensively Agoh and Dilcher [1, 2, 5] and the present author [9]. The Bernoulli numbers $B_{n}(n \geq 0)$ are defined by the generating function

$$
\frac{x}{e^{x}-1}=\sum_{n=0}^{\infty} B_{n} \frac{x^{n}}{n !} \quad(|x|<2 \pi) .
$$

2010 Mathematics Subject Classification: Primary 11B75; Secondary 05A15, 05 A40.

Keywords: Cauchy numbers of the second kind; convolution identities; Bernoulli numbers; Cauchy numbers of the first kind.

(C) 2015 Faculty of Mathematics, Kyushu University 
If we define

$$
\left(B_{l}+B_{m}\right)^{n}:=\sum_{j=0}^{n}\left(\begin{array}{c}
n \\
j
\end{array}\right) B_{l+j} B_{m+n-j},
$$

then the famous Euler's formula can be written as

$$
\left(B_{0}+B_{0}\right)^{n}=-n B_{n-1}-(n-1) B_{n} \quad(n \geq 1) .
$$

In [1] an expression for $\left(B_{l}+B_{m}\right)^{n}$ was found. In addition, some initial cases were listed, including, for example,

$$
\begin{aligned}
& \left(B_{0}+B_{1}\right)^{n}=-\frac{1}{2}(n+1) B_{n}-\frac{1}{2} n B_{n+1}, \\
& \left(B_{0}+B_{2}\right)^{n}=-\frac{1}{6}(n-1) B_{n}-\frac{1}{2} n B_{n+1}-\frac{1}{3} n B_{n+2}, \\
& \left(B_{1}+B_{1}\right)^{n}=\frac{1}{6}(n-1) B_{n}-B_{n+1}-\frac{1}{6}(n+3) B_{n+2} .
\end{aligned}
$$

The Cauchy numbers of the first kind $c_{n}(n \geq 0)$ are defined by

$$
c_{n}=\int_{0}^{1} x(x-1) \cdots(x-n+1) d x
$$

and the generating function of $c_{n}$ is given by

$$
\frac{x}{\ln (1+x)}=\sum_{n=0}^{\infty} c_{n} \frac{x^{n}}{n !} \quad(|x|<1)
$$

(see $[4,10]$ ). Note that the Cauchy numbers $c_{n}$ can be expressed in terms of the unsigned Stirling numbers of the first kind and $\left[\begin{array}{l}n \\ i\end{array}\right]$ :

$$
c_{n}=\sum_{i=0}^{n} \frac{(-1)^{n-i}}{i+1}\left[\begin{array}{l}
n \\
i
\end{array}\right]
$$

(see $[\mathbf{8}, \mathbf{1 0}]$ ), where the unsigned Stirling numbers of the first kind $\left[\begin{array}{c}n \\ i\end{array}\right]$ arise as coefficients of the rising factorial

$$
x(x+1) \cdots(x+n-1)=\sum_{i=0}^{n}\left[\begin{array}{l}
n \\
i
\end{array}\right] x^{i}
$$

(see, e.g., [7]). If we define $\left(c_{l}+c_{m}\right)^{n}$ by

$$
\left(c_{l}+c_{m}\right)^{n}:=\sum_{j=0}^{n}\left(\begin{array}{l}
n \\
j
\end{array}\right) c_{l+j} c_{m+n-j},
$$

an analogous formula to (1) is expressed by

$$
\left(c_{0}+c_{0}\right)^{n}=-n(n-2) c_{n-1}-(n-1) c_{n} \quad(n \geq 0)
$$

(see [12]). In [9], the present author gave a general explicit formula for $\left(c_{l}+c_{m}\right)^{n}(n \geq 0)$. Some initial cases include

$$
\begin{aligned}
& \left(c_{0}+c_{1}\right)^{n}=-\frac{1}{2}(n+1)(n-1) c_{n}-\frac{1}{2} n c_{n+1}, \\
& \left(c_{0}+c_{2}\right)^{n}=\frac{n !}{6} \sum_{k=0}^{n} \frac{(-1)^{n-k}(k-1) c_{k}}{k !}-\frac{1}{6} n(2 n+1) c_{n+1}-\frac{1}{3} n c_{n+2}, \\
& \left(c_{1}+c_{1}\right)^{n}=-\frac{n !}{6} \sum_{k=0}^{n} \frac{(-1)^{n-k}(k-1) c_{k}}{k !}-\frac{1}{6} n(n+5) c_{n+1}-\frac{1}{6}(n+3) c_{n+2} .
\end{aligned}
$$


In this paper we give expression formulae for $\left(\hat{c}_{l}+\hat{c}_{m}\right)^{n}$. Some of the initial cases are

$$
\begin{aligned}
\left(\hat{c}_{0}+\hat{c}_{0}\right)^{n}= & n ! \sum_{k=0}^{n}(-1)^{n-k} \frac{\hat{c}_{k}}{k !}-n \hat{c}_{n}, \\
\left(\hat{c}_{0}+\hat{c}_{1}\right)^{n}= & -\frac{(n+1) !}{2} \sum_{k=0}^{n}(-1)^{n-k} \frac{\hat{c}_{k}}{k !}-\frac{1}{2} n \hat{c}_{n+1}, \\
\left(\hat{c}_{0}+\hat{c}_{2}\right)^{n}= & \frac{n !}{12} \sum_{k=0}^{n} \frac{(-1)^{n-k}}{k !}(2 k(n+2 k-2)+5(n-k+2)(n-k+1)) \hat{c}_{k} \\
& -\frac{n}{3} \hat{c}_{n+2}-\frac{n}{2} \hat{c}_{n+1}, \\
\left(\hat{c}_{1}+\hat{c}_{1}\right)^{n}= & \frac{n !}{12} \sum_{k=0}^{n} \frac{(-1)^{n-k}}{k !}((n+1)(n+2)+k(8 n-9 k+19)) \hat{c}_{k}-\hat{c}_{n+1}-\frac{n+3}{6} \hat{c}_{n+2} .
\end{aligned}
$$

\section{Fundamental results}

Euler's identity (1) is an easy consequence of the formula

$$
b(x)^{2}=(1-x) b(x)-x b^{\prime}(x),
$$

where $b(x)=x /\left(e^{x}-1\right)$ (see, e.g., [6]). Similarly, the identity (3) is obtained because $c(x)=x / \ln (1+x)$ satisfies the formula

$$
c(x)^{2}=(1+x) c(x)-(1+x) x c^{\prime}(x) .
$$

Since $\hat{c}(x)=x /(1+x) \ln (1+x)$ satisfies the formula

$$
\hat{c}(x)^{2}=-x \hat{c}^{\prime}(x)+\frac{1}{1+x} \hat{c}(x),
$$

by $1 /(1+x)=\sum_{i=0}^{\infty}(-1)^{i} x^{i}$ and the fact

$$
x^{i} \hat{c}^{(v)}(x)=\sum_{n=0}^{\infty} \frac{n !}{(n-i) !} \hat{c}_{n+v-i} \frac{x^{n}}{n !} \quad(i, v \geq 0),
$$

we have

$$
\left(\hat{c}_{0}+\hat{c}_{0}\right)^{n}=-n \hat{c}_{n}+n ! \sum_{k=0}^{n}(-1)^{n-k} \frac{\hat{c}_{k}}{k !} \quad(n \geq 0),
$$

which is the formula (7). Differentiating both sides of (12) by $x$ and dividing them by 2 , we obtain

$$
\hat{c}(x) \hat{c}^{\prime}(x)=-\frac{1}{2} x \hat{c}^{\prime \prime}(x)-\frac{x}{2(1+x)} \hat{c}^{\prime}(x)-\frac{1}{2(1+x)^{2}} \hat{c}(x) .
$$

Then by (13) and for $k \geq 1$,

$$
\frac{1}{(1+x)^{k}}=\sum_{i=0}^{\infty}(-1)^{i}\left(\begin{array}{c}
k+i-1 \\
i
\end{array}\right) x^{i},
$$


we have

$$
\begin{aligned}
\sum_{n=0}^{\infty}\left(\hat{c}_{0}+\hat{c}_{1}\right)^{n} \frac{x^{n}}{n !}= & -\frac{1}{2} \sum_{n=0}^{\infty} n \hat{c}_{n+1} \frac{x^{n}}{n !}-\frac{1}{2} \sum_{i=0}^{\infty}(-1)^{i} \sum_{n=0}^{\infty} \frac{n !}{(n-i-1) !} \hat{c}_{n-i} \frac{x^{n}}{n !} \\
& -\frac{1}{2} \sum_{i=0}^{\infty}(-1)^{i}(i+1) \sum_{n=0}^{\infty} \frac{n !}{(n-i) !} \hat{c}_{n-i} \frac{x^{n}}{n !} \\
= & -\frac{1}{2} \sum_{n=0}^{\infty} n \hat{c}_{n+1} \frac{x^{n}}{n !}-\frac{1}{2} \sum_{i=0}^{n}(-1)^{i} \sum_{n=0}^{\infty} \frac{(n+1) !}{(n-i) !} \hat{c}_{n-i} \frac{x^{n}}{n !}
\end{aligned}
$$

yielding the formula (8).

In general, by differentiating both sides of (12) by $x$ at $\mu$ times, we have the following.

Proposition 1. For $\mu \geq 0$,

$$
\sum_{\kappa=0}^{\mu}\left(\begin{array}{l}
\mu \\
\kappa
\end{array}\right) \hat{c}^{(\kappa)}(x) \hat{c}^{(\mu-\kappa)}(x)=\sum_{\nu=0}^{\mu}(-1)^{\mu-\nu} \frac{\mu ! \hat{c}^{(\nu)}(x)}{\nu !(1+x)^{\mu-\nu+1}}-\mu \hat{c}^{(\mu)}(x)-x \hat{c}^{(\mu+1)}(x) .
$$

Proof. The left-hand side is due to the general Leibniz's rule. The right-hand side can be proved by induction. If we differentiate the right-hand side by $x$, then

$$
\begin{aligned}
& \sum_{\nu=0}^{\mu}\left((-1)^{\mu-v+1}(\mu-v+1) \frac{\mu ! \hat{c}^{(\nu)}(x)}{\nu !(1+x)^{\mu-\nu+2}}+(-1)^{\mu-v} \frac{\mu ! \hat{c}^{(\nu+1)}(x)}{\nu !(1+x)^{\mu-\nu+1}}\right) \\
& -\mu \hat{c}^{(\mu+1)}(x)-\hat{c}^{(\mu+1)}(x)-x \hat{c}^{(\mu+2)}(x) \\
& =\sum_{\nu=0}^{\mu}(-1)^{\mu-v+1} \frac{(\mu+1) ! \hat{c}^{(\nu)}(x)}{\nu !(1+x)^{\mu-v+2}}-\sum_{\nu=1}^{\mu}(-1)^{\mu-v+1} \frac{\mu ! \hat{c}^{(v)}(x)}{(\nu-1) !(1+x)^{\mu-v+2}} \\
& +\sum_{\nu=1}^{\mu+1}(-1)^{\mu-\nu+1} \frac{\mu ! \hat{c}^{(\nu)}(x)}{(\nu-1) !(1+x)^{\mu-\nu+2}}-(\mu+1) \hat{c}^{(\mu+1)}(x)-x \hat{c}^{(\mu+2)}(x) \\
& =\sum_{\nu=0}^{\mu+1}(-1)^{\mu-\nu+1} \frac{(\mu+1) ! \hat{c}^{(\nu)}(x)}{\nu !(1+x)^{\mu-\nu+2}}-(\mu+1) \hat{c}^{(\mu+1)}(x)-x \hat{c}^{(\mu+2)}(x) .
\end{aligned}
$$

By applying (15) and (13) in Proposition 1, we obtain the following result.

THEOREM 1. For $\mu \geq 0$ and $n \geq 0$ we have

$$
\sum_{\kappa=0}^{\mu}\left(\begin{array}{l}
\mu \\
\kappa
\end{array}\right)\left(\hat{c}_{\kappa}+\hat{c}_{\mu-\kappa}\right)^{n}=-(n+\mu) \hat{c}_{n+\mu}+\sum_{\nu=0}^{\mu} \frac{\mu ! n !}{\nu !} \sum_{i=0}^{n}(-1)^{\mu-v+i}\left(\begin{array}{c}
\mu-v+i \\
i
\end{array}\right) \frac{\hat{c}_{n+v-i}}{(n-i) !} .
$$

Examples. If we put $\mu=0,1$ in Theorem 1, we have the identities (7) and (8), respectively. If we put $\mu=2,3,4$ in Theorem 1 , we obtain

$$
\begin{aligned}
& \left(\hat{c}_{0}+\hat{c}_{2}\right)^{n}+\left(\hat{c}_{1}+\hat{c}_{1}\right)^{n}=\frac{(n+2) !}{2} \sum_{k=0}^{n}(-1)^{n-k} \frac{\hat{c}_{k}}{k !}-\frac{n+2}{2} \hat{c}_{n+1}-\frac{n+1}{2} \hat{c}_{n+2}, \\
& \left(\hat{c}_{0}+\hat{c}_{3}\right)^{n}+3\left(\hat{c}_{1}+\hat{c}_{2}\right)^{n} \\
& =-\frac{(n+3) !}{2} \sum_{k=0}^{n}(-1)^{n-k} \frac{\hat{c}_{k}}{k !}+\frac{(n+2)(n+3)}{2} \hat{c}_{n+1}-\frac{n+3}{2} \hat{c}_{n+2}-\frac{n+2}{2} \hat{c}_{n+3},
\end{aligned}
$$




$$
\begin{aligned}
\left(\hat{c}_{0}+\right. & \left.\hat{c}_{4}\right)^{n}+4\left(\hat{c}_{1}+\hat{c}_{3}\right)^{n}+3\left(\hat{c}_{2}+\hat{c}_{2}\right)^{n} \\
= & \frac{(n+4) !}{2} \sum_{k=0}^{n}(-1)^{n-k} \frac{\hat{c}_{k}}{k !}-\frac{(n+2)(n+3)(n+4)}{2} \hat{c}_{n+1}+\frac{(n+3)(n+4)}{2} \hat{c}_{n+2} \\
& -\frac{n+4}{2} \hat{c}_{n+3}-\frac{n+3}{2} \hat{c}_{n+4} .
\end{aligned}
$$

Next, note that $\hat{c}(x)$ satisfies the identity

$$
\hat{c}(x) \hat{c}^{\prime \prime}(x)=-\frac{x}{3} \hat{c}^{(3)}(x)-\frac{x}{2(1+x)} \hat{c}^{\prime \prime}(x)+\frac{x}{6(1+x)^{2}} \hat{c}^{\prime}(x)+\frac{5}{6(1+x)^{3}} \hat{c}(x) .
$$

By (13) and (15)

$$
\begin{aligned}
\left(\hat{c}_{0}+\hat{c}_{2}\right)^{n}= & -\frac{n}{3} \hat{c}_{n+2}-\frac{1}{2} \sum_{i=0}^{\infty}(-1)^{i} \frac{n !}{(n-i-1) !} \hat{c}_{n-i+1}+\frac{1}{6} \sum_{i=0}^{\infty}(-1)^{i} \frac{(i+1) n !}{(n-i-1) !} \hat{c}_{n-i} \\
& +\frac{5}{6} \sum_{i=0}^{\infty}(-1)^{i} \frac{(i+2)(i+1)}{2} \frac{n !}{(n-i) !} \hat{c}_{n-i} \\
= & -\frac{n}{3} \hat{c}_{n+2}-\frac{n}{2} \hat{c}_{n+1}+\frac{n !}{2} \sum_{i=0}^{n-2} \frac{(-1)^{i}}{(n-i-1) !} \hat{c}_{n-i} \\
& +\frac{n !}{6} \sum_{i=0}^{n-1} \frac{(-1)^{i}(i+1)}{(n-i-1) !} \hat{c}_{n-i}+\frac{5 n !}{12} \sum_{i=0}^{n-2} \frac{(-1)^{i}(i+2)(i+1)}{(n-i) !} \hat{c}_{n-i} \\
= & -\frac{n}{3} \hat{c}_{n+2}-\frac{n}{2} \hat{c}_{n+1}+\frac{n !}{12} \sum_{i=0}^{n} \frac{(-1)^{i}}{(n-i) !} \\
& \times(6(n-i)(n-i-1)+2(i+1)(n-i)+5(i+2)(i+1)) \hat{c}_{n-i},
\end{aligned}
$$

yielding the identity (9). In addition, by the identities (9) and (16), we have

$$
\begin{aligned}
\left(\hat{c}_{1}+\hat{c}_{1}\right)^{n}= & \frac{n !}{12} \sum_{k=0}^{n} \frac{(-1)^{n-k}}{k !}((n+1)(n+2)+k(8 n-9 k+19)) \hat{c}_{k}-\hat{c}_{n+1} \\
& -\frac{n+3}{6} \hat{c}_{n+2},
\end{aligned}
$$

which is the identity (10).

Proposition 2. For $m \geq 0$ we have

$$
\begin{aligned}
\hat{c}(x) \hat{c}^{(m)}(x)= & -\frac{x}{m+1} \hat{c}^{(m+1)}(x)-\sum_{k=0}^{m-1}\left(\begin{array}{l}
m \\
k
\end{array}\right) \frac{c_{k+1}}{k+1} \frac{x}{(1+x)^{k+1}} \hat{c}^{(m-k)}(x) \\
& +\frac{\hat{c}_{m}}{(1+x)^{m+1}} \hat{c}(x) .
\end{aligned}
$$

LEMMA 1. For $n \geq 0$ we have

$$
\hat{c}^{(n)}(x)=\left(\frac{-1}{1+x}\right)^{n+1} \sum_{j=0}^{n} \frac{\hat{g}_{n, j}}{(\ln (1+x))^{j+1}},
$$


where

$$
\hat{g}_{n, j}=j !\left(n\left[\begin{array}{c}
n \\
j+1
\end{array}\right]-\left[\begin{array}{l}
n \\
j
\end{array}\right] x\right) .
$$

Proof. Since $\hat{g}_{1,0}=1$ and $\hat{g}_{1,1}=-x$, for $n=1$ we have

$$
\begin{aligned}
\frac{d}{d x} \frac{x}{(1+x) \ln (1+x)} & =\frac{\ln (1+x)-x}{(1+x)^{2}(\ln (1+x))^{2}} \\
& =\frac{1}{(1+x)^{2}}\left(\frac{\hat{g}_{1,0}}{\ln (1+x)}+\frac{\hat{g}_{1,1}}{(\ln (1+x))^{2}}\right) .
\end{aligned}
$$

Assume that the result holds for $n$. Then

$$
\begin{aligned}
\frac{d^{n+1}}{d x^{n+1}} & \frac{x}{(1+x) \ln (1+x)} \\
= & (n+1)\left(\frac{-1}{1+x}\right)^{n+2} \sum_{j=0}^{n} \frac{\hat{g}_{n, j}}{(\ln (1+x))^{j+1}} \\
& +\left(\frac{-1}{1+x}\right)^{n+1} \sum_{j=0}^{n} \frac{-j !\left[\begin{array}{c}
n \\
j
\end{array}\right](\ln (1+x))^{j+1}-\hat{g}_{n, j}(j+1)(\ln (1+x))^{j}(1 /(1+x))}{(\ln (1+x))^{2 j+2}} \\
= & n\left(\frac{-1}{1+x}\right)^{n+2} \sum_{j=0}^{n} \frac{\hat{g}_{n, j}}{(\ln (1+x))^{j+1}}+\left(\frac{-1}{1+x}\right)^{n+2} \sum_{j=0}^{n} \frac{\hat{g}_{n, j}}{(\ln (1+x))^{j+1}} \\
& +\left(\frac{-1}{1+x}\right)^{n+2} \sum_{j=0}^{n} \frac{j !\left[\begin{array}{l}
n \\
j
\end{array}\right](1+x)}{(\ln (1+x))^{j+1}}+\left(\frac{-1}{1+x}\right)^{n+2} \sum_{j=0}^{n} \frac{\hat{g}_{n, j}(j+1)}{(\ln (1+x))^{j+2}} \\
= & \left(\frac{-1}{1+x}\right)^{n+2} \sum_{j=0}^{n} \frac{n \hat{g}_{n, j}+j !\left[\begin{array}{l}
n+1 \\
j+1
\end{array}\right]}{(\ln (1+x))^{j+1}}+\left(\frac{-1}{1+x}\right)^{n+2} \sum_{j=1}^{n+1} \frac{j^{j} \hat{g}_{n, j-1}}{(\ln (1+x))^{j+1}} \\
= & \left(\frac{-1}{1+x}\right)^{n+2} \sum_{j=0}^{n+1} \frac{\hat{g}_{n+1, j}}{(\ln (1+x))^{j+1}} .
\end{aligned}
$$

LEMMA 2. For integers $m$ and $j$ with $m \geq j \geq 0$ we have

$$
\hat{g}_{m+1, j}-(m+1) \hat{g}_{m, j-1}=\sum_{k=0}^{m-j}\left(\begin{array}{c}
m+1 \\
k+1
\end{array}\right)(-1)^{k} c_{k+1} \hat{g}_{m-k, j} .
$$

Proof. It is enough to prove

$$
\left[\begin{array}{c}
m+1 \\
j+1
\end{array}\right]-\frac{m}{j}\left[\begin{array}{c}
m \\
j
\end{array}\right]=\sum_{k=0}^{m-j}\left(\begin{array}{c}
m+1 \\
k+1
\end{array}\right)(-1)^{k} c_{k+1} \frac{m-k}{m+1}\left[\begin{array}{c}
m-k \\
j+1
\end{array}\right]
$$

and

$$
\left[\begin{array}{c}
m+1 \\
j
\end{array}\right]-\frac{m+1}{j}\left[\begin{array}{c}
m \\
j-1
\end{array}\right]=\sum_{k=0}^{m-j}\left(\begin{array}{c}
m+1 \\
k+1
\end{array}\right)(-1)^{k} c_{k+1}\left[\begin{array}{c}
m-k \\
j
\end{array}\right]
$$


The identity (20) holds because by (2) and [7, Table 241],

$$
\begin{aligned}
& \sum_{k=0}^{m-j+1}\left(\begin{array}{c}
m+1 \\
k
\end{array}\right)(-1)^{k} c_{k} \frac{m-k+1}{m+1}\left[\begin{array}{c}
m-k+1 \\
j+1
\end{array}\right] \\
& =\sum_{k=0}^{m-j+1}\left(\begin{array}{c}
m+1 \\
k
\end{array}\right)(-1)^{k} \sum_{i=0}^{k} \frac{(-1)^{k-i}}{i+1}\left[\begin{array}{c}
k \\
i
\end{array}\right] \frac{m-k+1}{m+1}\left[\begin{array}{c}
m-k+1 \\
j+1
\end{array}\right] \\
& =\sum_{i=0}^{m-j+1} \frac{(-1)^{i}}{i+1} \sum_{k=i}^{m-j+1}\left(\begin{array}{c}
m+1 \\
k
\end{array}\right)\left[\begin{array}{c}
k \\
i
\end{array}\right] \frac{m-k+1}{m+1}\left[\begin{array}{c}
m-k+1 \\
j+1
\end{array}\right] \\
& =\sum_{i=0}^{m-j+1} \frac{(-1)^{i}}{i+1}\left(\begin{array}{c}
i+j \\
j
\end{array}\right)\left[\begin{array}{c}
m+1 \\
i+j+1
\end{array}\right] \\
& =\frac{m}{j}\left[\begin{array}{c}
m \\
j
\end{array}\right] .
\end{aligned}
$$

Similarly, the identity (21) holds because

$$
\begin{aligned}
& \sum_{k=0}^{m-j+1}\left(\begin{array}{c}
m+1 \\
k
\end{array}\right)(-1)^{k} c_{k}\left[\begin{array}{c}
m-k+1 \\
j
\end{array}\right] \\
& =\sum_{k=0}^{m-j+1}\left(\begin{array}{c}
m+1 \\
k
\end{array}\right)(-1)^{k} \sum_{i=0}^{k} \frac{(-1)^{k-i}}{i+1}\left[\begin{array}{c}
k \\
i
\end{array}\right]\left[\begin{array}{c}
m-k+1 \\
j
\end{array}\right] \\
& =\sum_{i=0}^{m-j+1} \frac{(-1)^{i}}{i+1} \sum_{k=i}^{m-j+1}\left(\begin{array}{c}
m+1 \\
k
\end{array}\right)\left[\begin{array}{c}
k \\
i
\end{array}\right]\left[\begin{array}{c}
m-k+1 \\
j
\end{array}\right] \\
& =\sum_{i=0}^{m-j+1} \frac{(-1)^{i}}{i+1}\left(\begin{array}{c}
i+j \\
j
\end{array}\right)\left[\begin{array}{c}
m+1 \\
i+j
\end{array}\right] \\
& =\frac{m+1}{j}\left[\begin{array}{c}
m \\
j-1
\end{array}\right] .
\end{aligned}
$$

We also need the following relation [10, Theorem 2.4(2.1)] in order to prove Proposition 2.

LEMMA 3. For $m \geq 0$,

$$
(-1)^{m} \frac{\hat{c}_{m}}{m !}=\sum_{k=0}^{m}(-1)^{k} \frac{c_{k}}{k !} .
$$

Proof of Proposition 2. The identity (19) holds for $m=0$ and $m=1$ because of (12) and (14), respectively. Let $m \geq 2$. By Lemma 1 with $\hat{g}_{n, n}=-n ! x(n \geq 0), \hat{g}_{n, 0}=n !(n \geq 1)$ and $\hat{g}_{0,0}=-x$ together with Lemmata 2 and 3

$$
\begin{aligned}
\hat{c}(x) \hat{c}^{(m)}(x)+\frac{x}{m+1} \hat{c}^{(m+1)}(x) \\
\quad=\frac{x}{m+1}\left(\frac{-1}{1+x}\right)^{m+2}\left(\sum_{j=0}^{m-1} \frac{\left.\hat{g}_{m+1, j+1}-(m+1) \hat{g}_{m, j}\right)}{(\ln (1+x))^{j+2}}+\frac{(m+1) !}{\ln (1+x)}\right)
\end{aligned}
$$




$$
\begin{aligned}
= & \frac{x}{m+1}\left(\frac{-1}{1+x}\right)^{m+2}\left(\sum_{j=0}^{m-1} \frac{1}{(\ln (1+x))^{j+2}} \sum_{k=0}^{m-j-1}\left(\begin{array}{c}
m+1 \\
k+1
\end{array}\right)(-1)^{k} c_{k+1} \hat{g}_{m-k, j+1}\right. \\
& \left.+\frac{(m+1) !}{\ln (1+x)} \sum_{k=0}^{m-1}(-1)^{k} \frac{c_{k+1}}{(k+1) !}+\frac{(-1)^{m}(m+1) \hat{c}_{m}}{\ln (1+x)}\right) \\
= & \frac{x}{m+1}\left(\frac{-1}{1+x}\right)^{m+2}\left(\sum_{j=0}^{m} \frac{m+1}{(\ln (1+x))^{j+1}} \sum_{k=0}^{m-j} \frac{c_{k+1}}{k+1}\left(\begin{array}{l}
m \\
k
\end{array}\right)(-1)^{k} \hat{g}_{m-k, j}\right. \\
& \left.+\frac{(-1)^{m}}{\ln (1+x)}\left(c_{m+1} x+(m+1) \hat{c}_{m}\right)\right) \\
= & \left(\frac{-1}{1+x}\right)^{m+2} \sum_{k=0}^{m}\left(\begin{array}{l}
m \\
k
\end{array}\right) \frac{c_{k+1}}{k+1}(-1)^{k} \sum_{j=0}^{m-k} \frac{x \hat{g}_{m-k, j}}{(\ln (1+x))^{j+1}} \\
& +\left(\frac{-1}{1+x}\right)^{m+2} \frac{x}{\ln (1+x)}(-1)^{m}\left(\frac{c_{m+1}}{m+1} x+\hat{c}_{m}\right) \\
= & -\sum_{k=0}^{m-1}\left(\begin{array}{l}
m \\
k
\end{array}\right) \frac{c_{k+1}}{k+1} \frac{x}{(1+x)^{k+1}} \hat{c}^{(m-k)}(x)+\frac{\hat{c}_{m}}{(1+x)^{m+1}} \hat{c}(x) .
\end{aligned}
$$

By Proposition 2, we obtain the following theorem.

THEOREM 2. For $m \geq 0$ and $n \geq 0$ we have

$$
\begin{aligned}
\left(\hat{c}_{0}+\hat{c}_{m}\right)^{n}= & -\frac{n}{m+1} \hat{c}_{n+m} \\
& -\frac{1}{m+1} \sum_{k=0}^{m-1}\left(\begin{array}{c}
m+1 \\
k+1
\end{array}\right) c_{k+1} \sum_{i=0}^{n-1}(-1)^{i}\left(\begin{array}{c}
k+i \\
i
\end{array}\right) \frac{n !}{(n-i-1) !} \hat{c}_{n+m-k-i-1} \\
& +\hat{c}_{m} \sum_{i=0}^{n}(-1)^{i}\left(\begin{array}{c}
m+i \\
i
\end{array}\right) \frac{n !}{(n-i) !} \hat{c}_{n-i} .
\end{aligned}
$$

Examples. When $m=0, m=1$ and $m=2$, we have the formulae (7), (8) and (9), respectively. If $m=3$, we have

$$
\begin{aligned}
\left(\hat{c}_{0}+\hat{c}_{3}\right)^{n}= & -\frac{(n+1) !}{8} \sum_{k=0}^{n} \frac{(-1)^{n-k}\left(2\left(2 n^{2}-6 n+9\right)-(n-k)(n+9 k-27)\right) \hat{c}_{k}}{k !} \\
& +\frac{n(2 n-1) \hat{c}_{n+1}}{4}-\frac{n \hat{c}_{n+2}}{2}-\frac{n \hat{c}_{n+3}}{4} .
\end{aligned}
$$

Proof. By (13),

$$
x \hat{c}^{(m+1)}(x)=\sum_{n=0}^{\infty} n \hat{c}_{n+m} \frac{x^{n}}{n !} .
$$


By (15) we get

$$
\begin{aligned}
& \sum_{k=0}^{m-1}\left(\begin{array}{c}
m+1 \\
k+1
\end{array}\right) \frac{c_{k+1} x}{(1+x)^{k+1}} \hat{c}^{(m-k)}(x) \\
& \quad=\sum_{k=0}^{m-1}\left(\begin{array}{c}
m+1 \\
k+1
\end{array}\right) c_{k+1} \sum_{i=0}^{\infty}(-1)^{i}\left(\begin{array}{c}
k+i \\
i
\end{array}\right) x^{i+1} \hat{c}^{(m-k)}(x) \\
& \quad=\sum_{k=0}^{m-1}\left(\begin{array}{c}
m+1 \\
k+1
\end{array}\right) c_{k+1} \sum_{i=0}^{n-1}(-1)^{i}\left(\begin{array}{c}
k+i \\
i
\end{array}\right) \sum_{n=0}^{\infty} \frac{n !}{(n-i-1) !} \hat{c}_{n+m-k-i-1} \frac{x^{n}}{n !}
\end{aligned}
$$

Finally, for $m \geq 0$

$$
\frac{\hat{c}_{m}}{(1+x)^{m+1}} \hat{c}(x)=\hat{c}_{m} \sum_{i=0}^{n}(-1)^{i}\left(\begin{array}{c}
m+i \\
i
\end{array}\right) \sum_{n=0}^{\infty} \frac{n !}{(n-i) !} \hat{c}_{n-i} \frac{x^{n}}{n !}
$$

Proposition 3. For $m \geq 1$ we have

$$
\begin{aligned}
\hat{c}^{\prime}(x) \hat{c}^{(m)}(x)= & -\frac{x}{(m+2)(m+1)} \hat{c}^{(m+2)}(x)-\frac{1}{m+1} \hat{c}^{(m+1)}(x) \\
& +\sum_{k=0}^{m-2}\left(\begin{array}{l}
m \\
k
\end{array}\right)\left(\frac{c_{k+2}}{k+2}+\frac{k c_{k+1}}{k+1}\right) \frac{x \hat{c}^{(m-k)}(x)}{(1+x)^{k+2}} \\
& -\sum_{k=0}^{m-2}\left(\begin{array}{l}
m \\
k
\end{array}\right) \frac{c_{k+1}}{k+1} \frac{\hat{c}^{(m-k)}(x)}{(1+x)^{k+2}} \\
& +\frac{\left(c_{m+1}+(m-1) c_{m}\right) x+\left(\hat{c}_{m}-c_{m}\right)}{(1+x)^{m+1}} \hat{c}^{\prime}(x)-\frac{\hat{c}_{m+1}+(m+1) \hat{c}_{m}}{(1+x)^{m+2}} \hat{c}(x) .
\end{aligned}
$$

Proof. By Proposition 2 for $m \geq 1$ we have

$$
\begin{aligned}
\hat{c}^{\prime}(x) \hat{c}^{(m)}(x)= & \left(\hat{c}(x) \hat{c}^{(m)}(x)\right)^{\prime}-\hat{c}(x) \hat{c}^{(m+1)} \\
= & -\frac{1}{m+1} \hat{c}^{(m+1)}(x)-\frac{x}{m+1} \hat{c}^{(m+2)}(x) \\
& +\sum_{k=0}^{m-1}\left(\begin{array}{c}
m \\
k
\end{array}\right) \frac{k c_{k+1}}{k+1} \frac{x}{(1+x)^{k+2}} \hat{c}^{(m-k)}(x) \\
& -\sum_{k=0}^{m-1}\left(\begin{array}{c}
m \\
k
\end{array}\right) \frac{c_{k+1}}{k+1} \frac{1}{(1+x)^{k+2}} \hat{c}^{(m-k)}(x) \\
& -\sum_{k=0}^{m-1}\left(\begin{array}{c}
m \\
k
\end{array}\right) \frac{c_{k+1}}{k+1} \frac{x}{(1+x)^{k+1}} \hat{c}^{(m-k+1)}(x) \\
& -\frac{(m+1) \hat{c}_{m}}{(1+x)^{m+2}} \hat{c}(x)+\frac{\hat{c}_{m}}{(1+x)^{m+1}} \hat{c}^{\prime}(x)
\end{aligned}
$$




$$
\begin{aligned}
& +\frac{x}{m+2} \hat{c}^{(m+2)}(x)+\sum_{k=0}^{m}\left(\begin{array}{c}
m+1 \\
k
\end{array}\right) \frac{c_{k+1}}{k+1} \frac{x}{(1+x)^{k+1}} \hat{c}^{(m-k+1)}(x) \\
& -\frac{\hat{c}_{m+1}}{(1+x)^{m+2}} \hat{c}(x) \\
& =-\frac{x}{(m+2)(m+1)} \hat{c}^{(m+2)}(x)-\frac{1}{m+1} \hat{c}^{(m+1)}(x) \\
& +\sum_{k=0}^{m-2}\left(\begin{array}{c}
m \\
k
\end{array}\right) \frac{k c_{k+1}}{k+1} \frac{x}{(1+x)^{k+2}} \hat{c}^{(m-k)}(x)+(m-1) c_{m} \frac{x}{(1+x)^{m+1}} \hat{c}^{\prime}(x) \\
& -\sum_{k=0}^{m-2}\left(\begin{array}{c}
m \\
k
\end{array}\right) \frac{c_{k+1}}{k+1} \frac{1}{(1+x)^{k+2}} \hat{c}^{(m-k)}(x)-\frac{c_{m}}{(1+x)^{m+1}} \hat{c}^{\prime}(x) \\
& -\sum_{k=1}^{m-1}\left(\begin{array}{l}
m \\
k
\end{array}\right) \frac{c_{k+1}}{k+1} \frac{x}{(1+x)^{k+1}} \hat{c}^{(m-k+1)}(x) \\
& +\sum_{k=1}^{m-1}\left(\begin{array}{c}
m+1 \\
k
\end{array}\right) \frac{c_{k+1}}{k+1} \frac{x}{(1+x)^{k+1}} \hat{c}^{(m-k+1)}(x)+\frac{c_{m+1} x}{(1+x)^{m+1}} \hat{c}^{\prime}(x) \\
& +\frac{\hat{c}_{m}}{(1+x)^{m+1}} \hat{c}^{\prime}(x)-\frac{\hat{c}_{m+1}+(m+1) \hat{c}_{m}}{(1+x)^{m+2}} \hat{c}(x) \\
& =-\frac{x}{(m+2)(m+1)} \hat{c}^{(m+2)}(x)-\frac{1}{m+1} \hat{c}^{(m+1)}(x) \\
& +\sum_{k=0}^{m-2}\left(\begin{array}{l}
m \\
k
\end{array}\right)\left(\frac{c_{k+2}}{k+2}+\frac{k c_{k+1}}{k+1}\right) \frac{x \hat{c}^{(m-k)}(x)}{(1+x)^{k+2}} \\
& -\sum_{k=0}^{m-2}\left(\begin{array}{c}
m \\
k
\end{array}\right) \frac{c_{k+1}}{k+1} \frac{\hat{c}^{(m-k)}(x)}{(1+x)^{k+2}} \\
& +\frac{\left(c_{m+1}+(m-1) c_{m}\right) x+\left(\hat{c}_{m}-c_{m}\right)}{(1+x)^{m+1}} \hat{c}^{\prime}(x)-\frac{\hat{c}_{m+1}+(m+1) \hat{c}_{m}}{(1+x)^{m+2}} \hat{c}(x) .
\end{aligned}
$$

By Proposition 3 we have the following.

THEOREM 3. For $m \geq 1$ and $n \geq 0$ we have

$$
\begin{aligned}
\left(\hat{c}_{1}+\right. & \left.\hat{c}_{m}\right)^{n} \\
= & -\frac{(n+m+2) \hat{c}_{n+m+1}}{(m+2)(m+1)} \\
& +\sum_{k=0}^{m-2}\left(\begin{array}{l}
m \\
k
\end{array}\right)\left(\frac{c_{k+2}}{k+2}+\frac{k c_{k+1}}{k+1}\right) \sum_{i=0}^{n-1}(-1)^{i}\left(\begin{array}{c}
k+i+1 \\
i
\end{array}\right) \frac{n !}{(n-i-1) !} \hat{c}_{n+m-k-i-1} \\
& -\sum_{k=0}^{m-2}\left(\begin{array}{c}
m \\
k
\end{array}\right) \frac{c_{k+1}}{k+1} \sum_{i=0}^{n}(-1)^{i}\left(\begin{array}{c}
k+i+1 \\
i
\end{array}\right) \frac{n !}{(n-i) !} \hat{c}_{n+m-k-i} \\
& +\left(c_{m+1}+(m-1) c_{m}\right) \sum_{i=0}^{n-1}(-1)^{i}\left(\begin{array}{c}
m+i \\
i
\end{array}\right) \frac{n !}{(n-i-1) !} \hat{c}_{n-i}
\end{aligned}
$$




$$
\begin{aligned}
& +\left(\hat{c}_{m}-c_{m}\right) \sum_{i=0}^{n}(-1)^{i}\left(\begin{array}{c}
m+i \\
i
\end{array}\right) \frac{n !}{(n-i) !} \hat{c}_{n-i+1} \\
& -\left(\hat{c}_{m+1}+(m+1) \hat{c}_{m}\right) \sum_{i=0}^{n}(-1)^{i}\left(\begin{array}{c}
m+i+1 \\
i
\end{array}\right) \frac{n !}{(n-i) !} \hat{c}_{n-i} .
\end{aligned}
$$

Examples. If $m=1$, we have the identity (10). If $m=2$, we have

$$
\begin{aligned}
\left(\hat{c}_{1}+\hat{c}_{2}\right)^{n}= & -\frac{(n+1) !}{24} \sum_{k=0}^{n} \frac{(-1)^{n-k} \hat{c}_{k}}{k !}((n+2)(n+3)+k(8 n-9 k+27)) \\
& +\frac{(11 n+12) \hat{c}_{n+1}}{12}-\frac{\hat{c}_{n+2}}{2}-\frac{(n+4) \hat{c}_{n+3}}{12} .
\end{aligned}
$$

By $\hat{c}^{\prime \prime}(x) \hat{c}^{(m)}(x)=\left(\hat{c}^{\prime}(x) \hat{c}^{(m)}(x)\right)^{\prime}-\hat{c}^{\prime}(x) \hat{c}^{(m+1)}(x)$ in Proposition 3, we have the following.

Proposition 4. For $m \geq 2$ we have

$$
\begin{aligned}
& \hat{c}^{\prime \prime}(x) \hat{c}^{(m)}(x) \\
&=- \frac{2 x}{(m+3)(m+2)(m+1)} \hat{c}^{(m+3)}(x)-\frac{2}{(m+2)(m+1)} \hat{c}^{(m+2)}(x) \\
&-\sum_{k=0}^{m-3}\left(\begin{array}{l}
m \\
k
\end{array}\right)\left(\frac{c_{k+3}}{k+3}+\frac{2(k+1) c_{k+2}}{k+2}+k c_{k+1}\right) \frac{x}{(1+x)^{k+3}} \hat{c}^{(m-k)}(x) \\
&+2 \sum_{k=0}^{m-3}\left(\begin{array}{l}
m \\
k
\end{array}\right)\left(\frac{c_{k+2}}{k+2}+c_{k+1}\right) \frac{1}{(1+x)^{k+3}} \hat{c}^{(m-k)}(x) \\
&-\frac{1}{2}\left((m-2) c_{m+1}+2(m-1)^{2} c_{m}+m(m-1)(m-2) c_{m-1}\right) \frac{x}{(1+x)^{m+1}} \hat{c}^{\prime \prime}(x) \\
&+\left((m-1) c_{m}+m(m-1) c_{m-1}+\hat{c}_{m}\right) \frac{1}{(1+x)^{m+1}} \hat{c}^{\prime \prime}(x) \\
&-\left(c_{m+2}+2 m c_{m+1}+m(m-1) c_{m}\right) \frac{x}{(1+x)^{m+2}} \hat{c}^{\prime}(x) \\
&+2\left(c_{m+1}+m c_{m}-\hat{c}_{m+1}-(m+1) \hat{c}_{m}\right) \frac{1}{(1+x)^{m+2}} \hat{c}^{\prime}(x) \\
&+\left(\hat{c}_{m+2}+2(m+2) \hat{c}_{m+1}+(m+2)(m+1) \hat{c}_{m}\right) \frac{1}{(1+x)^{m+3}} \hat{c}(x) .
\end{aligned}
$$

By Proposition 4, we have the following result.

THEOREM 4. For $m \geq 2$ and $n \geq 0$,

$$
\begin{aligned}
\left(\hat{c}_{2}\right. & \left.+\hat{c}_{m}\right)^{n} \\
& =-\frac{2(n+m+3)}{(m+3)(m+2)(m+1)} \hat{c}_{n+m+2}
\end{aligned}
$$




$$
\begin{aligned}
& -\sum_{k=0}^{m-3}\left(\begin{array}{c}
m \\
k
\end{array}\right)\left(\frac{c_{k+3}}{k+3}+\frac{2(k+1) c_{k+2}}{k+2}+k c_{k+1}\right) \\
& \times \sum_{i=0}^{n-1}(-1)^{i}\left(\begin{array}{c}
k+i+2 \\
i
\end{array}\right) \frac{n !}{(n-i-1) !} \hat{c}_{n+m-k-i-1} \\
& +2 \sum_{k=0}^{m-3}\left(\begin{array}{l}
m \\
k
\end{array}\right)\left(\frac{c_{k+2}}{k+2}+c_{k+1}\right) \sum_{i=0}^{n}(-1)^{i}\left(\begin{array}{c}
k+i+2 \\
i
\end{array}\right) \frac{n !}{(n-i) !} \hat{c}_{n+m-k-i} \\
& -\frac{1}{2}\left((m-2) c_{m+1}+2(m-1)^{2} c_{m}+m(m-1)(m-2) c_{m-1}\right) \\
& \times \sum_{i=0}^{n-1}(-1)^{i}\left(\begin{array}{c}
m+i \\
i
\end{array}\right) \frac{n !}{(n-i-1) !} \hat{c}_{n-i+1} \\
& +\left((m-1) c_{m}+m(m-1) c_{m-1}+\hat{c}_{m}\right) \sum_{i=0}^{n}(-1)^{i}\left(\begin{array}{c}
m+i \\
i
\end{array}\right) \frac{n !}{(n-i) !} \hat{c}_{n-i+2} \\
& -\left(c_{m+2}+2 m c_{m+1}+m(m-1) c_{m}\right) \sum_{i=0}^{n-1}(-1)^{i}\left(\begin{array}{c}
m+i+1 \\
i
\end{array}\right) \frac{n !}{(n-i-1) !} \hat{c}_{n-i} \\
& +2\left(c_{m+1}+m c_{m}-\hat{c}_{m+1}-(m+1) \hat{c}_{m}\right) \sum_{i=0}^{n}(-1)^{i}\left(\begin{array}{c}
m+i+1 \\
i
\end{array}\right) \frac{n !}{(n-i) !} \hat{c}_{n-i+1} \\
& +\left(\hat{c}_{m+2}+2(m+2) \hat{c}_{m+1}+(m+2)(m+1) \hat{c}_{m}\right) \sum_{i=0}^{n}(-1)^{i}\left(\begin{array}{c}
m+i+2 \\
i
\end{array}\right) \frac{n !}{(n-i) !} \hat{c}_{n-i} .
\end{aligned}
$$

Example. If $m=2$, we have

$$
\begin{aligned}
\left(\hat{c}_{2}+\hat{c}_{2}\right)^{n}= & \frac{n !}{6 !} \sum_{k=0}^{n} \frac{\hat{c}_{k}}{k !}(-1)^{n-k}(n-k+3)(11(n-k+4)(n-k+2)(n-k+1) \\
& +4 k(15(9 n-9 k+38)(k-1)+(19 n-19 k+79)(n-k+2))) \\
& -\frac{(29 n+4) \hat{c}_{n+1}}{6}+\frac{5 \hat{c}_{n+2}}{3}-\frac{(n+5) \hat{c}_{n+4}}{30} .
\end{aligned}
$$

\section{The general case}

For general integers $l$ and $m$, we obtain the following result.

Proposition 5. Let $l, m$ be fixed non-negative integers with $m \geq l \geq 1$. Then

$$
\begin{aligned}
\hat{c}^{(l)}(x) \hat{c}^{(m)}(x)= & -\frac{l ! m !}{(l+m+1) !} x \hat{c}^{(l+m+1)}(x)-\frac{l ! m !}{(l+m) !} \hat{c}^{(l+m)}(x) \\
& +\sum_{k=0}^{m-l-1} \frac{a_{l, m \cdot m-k} x+b_{l, m \cdot m-k}}{(1+x)^{l+k+1}} \hat{c}^{(m-k)}(x) \\
& +\sum_{k=0}^{l} \frac{a_{l, m, l-k} x+b_{l, m, l-k}}{(1+x)^{m+k+1}} \hat{c}^{(l-k)}(x),
\end{aligned}
$$


where for $l+1 \leq r=m-k \leq m$,

$$
\begin{aligned}
& a_{l, m, r}=(-1)^{l+1}\left(\begin{array}{c}
m \\
k
\end{array}\right) \sum_{i=0}^{l} i !\left(\begin{array}{c}
l \\
i
\end{array}\right)\left(\begin{array}{c}
l+k-1 \\
i
\end{array}\right) \frac{c_{l+k-i+1}}{l+k-i+1}, \\
& b_{l, m, r}=(-1)^{l} l\left(\begin{array}{c}
m \\
k
\end{array}\right) \sum_{i=0}^{l-1} i !\left(\begin{array}{c}
l-1 \\
i
\end{array}\right)\left(\begin{array}{c}
l+k-1 \\
i
\end{array}\right) \frac{c_{l+k-i}}{l+k-i},
\end{aligned}
$$

and for $1 \leq r=l-k \leq l$,

$$
\begin{aligned}
a_{l, m, r}= & \sum_{j=0}^{r-2} \frac{(-1)^{l+j+1}}{r}\left(\begin{array}{l}
l \\
j
\end{array}\right)\left(\begin{array}{c}
m \\
r-j-1
\end{array}\right)\left(\begin{array}{c}
r-1 \\
j
\end{array}\right)^{-1} \\
& \times \sum_{i=0}^{l-j} i !\left(\begin{array}{c}
l-j \\
i
\end{array}\right)\left(\begin{array}{c}
l+m-r-1 \\
i
\end{array}\right) c_{l+m-i-r+1} \\
& +\frac{(-1)^{l+r}}{r}\left(\begin{array}{c}
l \\
r-1
\end{array}\right) \sum_{i=0}^{l-r+1} i !\left(\begin{array}{c}
l-r+1 \\
i
\end{array}\right)\left(\begin{array}{c}
l+m-r-1 \\
i
\end{array}\right) c_{l+m-i-r+1}
\end{aligned}
$$

with $a_{l, m, 0}=0$ and for $0 \leq r=l-k \leq l$,

$$
\begin{aligned}
b_{l, m, r}= & \sum_{j=1}^{r}(-1)^{l+j+1}\left(\begin{array}{l}
l \\
j
\end{array}\right)\left(\begin{array}{c}
m \\
r-j
\end{array}\right)\left(\begin{array}{l}
r \\
j
\end{array}\right)^{-1} \sum_{i=0}^{l-j} i !\left(\begin{array}{c}
l-j \\
i
\end{array}\right)\left(\begin{array}{c}
l+m-r-1 \\
i
\end{array}\right) c_{l+m-i-r} \\
& +(-1)^{l+r}\left(\begin{array}{l}
l \\
r
\end{array}\right) \sum_{i=0}^{l-r} i !\left(\begin{array}{c}
l-r \\
i
\end{array}\right)\left(\begin{array}{c}
l+m-r \\
i
\end{array}\right) \hat{c}_{l+m-i-r .}
\end{aligned}
$$

Proof. Note that $a_{l, m+1, m+1}=a_{l, m, m}, b_{l, m+1, m+1}=b_{l, m, m}$, and for $k=0,1, \ldots, m-l-1$,

$$
\begin{aligned}
-(l+k) a_{l, m, m-k}+a_{l, m, m-k-1}-a_{l, m+1, m-k} & =a_{l+1, m, m-k}, \\
a_{l, m, m-k}-(l+k+1) b_{l, m, m-k}-b_{l, m+1, m-k}+b_{l, m, m-k-1} & =b_{l+1, m, m-k},
\end{aligned}
$$

and for $k=0,1, \ldots, l-1$,

$$
\begin{aligned}
-(m+k) a_{l, m, l-k}+a_{l, m, l-k-1}-a_{l, m+1, l-k} & =a_{l+1, m, l-k}, \\
a_{l, m, l-k}-(m+k+1) b_{l, m, l-k}+b_{l, m, l-k-1}-b_{l, m+1, l-k} & =b_{l+1, m, l-k},
\end{aligned}
$$

and $-(l+m+1) b_{l, m, 0}-b_{l, m+1,0}=b_{l+1, m, 0}$. By using these relations, we have

$$
\begin{aligned}
\left(\hat{c}^{(l)}(x) \hat{c}^{(m)}(x)\right)^{\prime}-\hat{c}^{(l)}(x) \hat{c}^{(m+1)}(x) & \\
= & -\frac{l ! m !}{(l+m+1) !} \hat{c}^{(l+m+1)}(x)-\frac{l ! m !}{(l+m+1) !} x \hat{c}^{(l+m+2)}(x)-\frac{l ! m !}{(l+m) !} \hat{c}^{(l+m+1)}(x) \\
& +\frac{l !(m+1) !}{(l+m+2) !} x \hat{c}^{(l+m+2)}(x)+\frac{l !(m+1) !}{(l+m+1) !} \hat{c}^{(l+m+1)}(x) \\
& -\sum_{k=0}^{m-l-1}(l+k) \frac{a_{l, m, m-k} x}{(1+x)^{l+k+2}} \hat{c}^{(m-k)}(x)+\sum_{k=0}^{m-l-2} \frac{a_{l, m, m-k-1} x}{(1+x)^{l+k+2}} \hat{c}^{(m-k)}(x)
\end{aligned}
$$




$$
\begin{aligned}
& -\sum_{k=0}^{m-l-1} \frac{a_{l, m+1, m-k} x}{(1+x)^{l+k+2}} \hat{c}^{(m-k)}(x)-\sum_{k=0}^{m-l-1}(l+k+1) \frac{b_{l, m, m-k}}{(1+x)^{l+k+2}} \hat{c}^{(m-k)}(x) \\
& +\sum_{k=0}^{m-l-2} \frac{b_{l, m, m-k-1}}{(1+x)^{l+k+2}} \hat{c}^{(m-k)}(x)+\sum_{k=0}^{m-l-1} \frac{a_{l, m, m-k}}{(1+x)^{l+k+2}} \hat{c}^{(m-k)}(x) \\
& -\sum_{k=0}^{m-l-1} \frac{b_{l, m+1, m-k}}{(1+x)^{l+k+2}} \hat{c}^{(m-k)}(x)-\sum_{k=0}^{l}(m+k) \frac{a_{l, m, l-k} x}{(1+x)^{m+k+2}} \hat{c}^{(l-k)}(x) \\
& +\sum_{k=0}^{l-1} \frac{a_{l, m, l-k-1} x}{(1+x)^{m+k+2}} \hat{c}^{(l-k)}(x)-\sum_{k=0}^{l} \frac{a_{l, m+1, l-k} x}{(1+x)^{m+k+2}} \hat{c}^{(l-k)}(x) \\
& -\sum_{k=0}^{l}(m+k+1) \frac{b_{l, m, l-k}}{(1+x)^{m+k+2}} \hat{c}^{(l-k)}(x)+\sum_{k=0}^{l-1} \frac{b_{l, m, l-k-1}}{(1+x)^{m+k+2}} \hat{c}^{(l-k)}(x) \\
& +\sum_{k=0}^{l} \frac{a_{l, m, l-k}}{(1+x)^{m+k+2}} \hat{c}^{(l-k)}(x)-\sum_{k=0}^{l} \frac{b_{l, m+1, l-k}}{(1+x)^{m+k+2}} \hat{c}^{(l-k)}(x) \\
& +\frac{a_{l, m, l} x+b_{l, m, l}}{(1+x)^{m+1}} \hat{c}^{(l+1)}(x) \\
& =-\frac{(l+1) ! m !}{(l+m+2) !} x \hat{c}^{(l+m+2)}(x)-\frac{(l+1) ! m !}{(l+m+1) !} \hat{c}^{(l+m+1)}(x) \\
& +\sum_{k=0}^{m-l-2} \frac{a_{l+1, m, m-k} x}{(1+x)^{l+k+2}} \hat{c}^{(m-k)}(x)+\sum_{k=0}^{m-l-2} \frac{b_{l+1, m, m-k}}{(1+x)^{l+k+2}} \hat{c}^{(m-k)}(x) \\
& -(m-1) \frac{a_{l, m, l+1} x}{(1+x)^{m+1}} \hat{c}^{(l+1)}(x)-\frac{a_{l, m+1, l+1} x}{(1+x)^{m+1}} \hat{c}^{(l+1)}(x) \\
& +\frac{a_{l, m, l+1}}{(1+x)^{m+1}} \hat{c}^{(l+1)}(x)-m \frac{b_{l, m, l+1} x}{(1+x)^{m+1}} \hat{c}^{(l+1)}(x)-\frac{b_{l, m+1, l+1} x}{(1+x)^{m+1}} \hat{c}^{(l+1)}(x) \\
& +\frac{a_{l, m, l} x+b_{l, m, l}}{(1+x)^{m+1}} \hat{c}^{(l+1)}(x)+\sum_{k=0}^{l-1} \frac{a_{l+1, m, l-k} x}{(1+x)^{m+k+2}} \hat{c}^{(l-k)}(x) \\
& +\sum_{k=0}^{l-1} \frac{b_{l+1, m, l-k}}{(1+x)^{m+k+2}} \hat{c}^{(l-k)}(x)+\frac{b_{l+1, m, 0}}{(1+x)^{l+m+2}} \hat{c}(x) \\
& =-\frac{(l+1) ! m !}{(l+m+2) !} x \hat{c}^{(l+m+2)}(x)-\frac{(l+1) ! m !}{(l+m+1) !} \hat{c}^{(l+m+1)}(x) \\
& +\sum_{k=0}^{m-l-2} \frac{a_{l+1, m, m-k} x}{(1+x)^{l+k+2}} \hat{c}^{(m-k)}(x)+\sum_{k=0}^{m-l-2} \frac{b_{l+1, m, m-k}}{(1+x)^{l+k+2}} \hat{c}^{(m-k)}(x) \\
& +\sum_{k=1}^{l+1} \frac{a_{l+1, m, l-k+1} x}{(1+x)^{m+k+1}} \hat{c}^{(l-k+1)}(x)+\sum_{k=1}^{l+1} \frac{b_{l+1, m, l-k+1}}{(1+x)^{m+k+1}} \hat{c}^{(l-k+1)}(x) \\
& +\frac{a_{l+1, m, l+1} x+b_{l+1, m, l+1}}{(1+x)^{m+1}} \hat{c}^{(l+1)}(x) \\
& =\hat{c}^{(l+1)}(x) \hat{c}^{(m)}(x) \text {. }
\end{aligned}
$$


Finally, we shall prove only the relations (24) and (25). Other relations can be proved similarly and omitted. The left-hand side of (24) is equal to

$$
\begin{aligned}
& -(l+k)(-1)^{l+1}\left(\begin{array}{l}
m \\
k
\end{array}\right) \sum_{i=0}^{l} i !\left(\begin{array}{l}
l \\
i
\end{array}\right)\left(\begin{array}{c}
l+k-1 \\
i
\end{array}\right) \frac{c_{l+k-i+1}}{l+k-i+1} \\
& +(-1)^{l+1}\left(\begin{array}{c}
m \\
k+1
\end{array}\right) \sum_{i=0}^{l} i !\left(\begin{array}{l}
l \\
i
\end{array}\right)\left(\begin{array}{c}
l+k \\
i
\end{array}\right) \frac{c_{l+k-i+2}}{l+k-i+2} \\
& -(-1)^{l+1}\left(\begin{array}{c}
m+1 \\
k+1
\end{array}\right) \sum_{i=0}^{l} i !\left(\begin{array}{l}
l \\
i
\end{array}\right)\left(\begin{array}{c}
l+k \\
i
\end{array}\right) \frac{c_{l+k-i+2}}{l+k-i+2} \\
& =-(l+k)(-1)^{l+1}\left(\begin{array}{c}
m \\
k
\end{array}\right) \sum_{i=1}^{l+1}(i-1) !\left(\begin{array}{c}
l \\
i-1
\end{array}\right)\left(\begin{array}{c}
l+k-1 \\
i-1
\end{array}\right) \frac{c_{l+k-i+2}}{l+k-i+2} \\
& -(-1)^{l+1}\left(\begin{array}{c}
m \\
k
\end{array}\right) \sum_{i=0}^{l} i !\left(\begin{array}{c}
l \\
i
\end{array}\right)\left(\begin{array}{c}
l+k \\
i
\end{array}\right) \frac{c_{l+k-i+2}}{l+k-i+2} \\
& =(-1)^{l}\left(\begin{array}{c}
m \\
k
\end{array}\right) \sum_{i=0}^{l+1} i !\left(\begin{array}{c}
l+1 \\
i
\end{array}\right)\left(\begin{array}{c}
l+k \\
i
\end{array}\right) \frac{c_{l+k-i+2}}{l+k-i+2} \\
& =a_{l+1, m, m-k} \text {. }
\end{aligned}
$$

The left-hand side of (25) is equal to

$$
\begin{aligned}
& -(l+m-r-1) a_{l, m, r}-a_{l, m+1, r}+a_{l, m, r-1} \\
& =-(l+m-r-1) \sum_{j=0}^{r-2} \frac{(-1)^{l+j+1}}{r}\left(\begin{array}{l}
l \\
j
\end{array}\right)\left(\begin{array}{c}
m \\
r-j-1
\end{array}\right)\left(\begin{array}{c}
r-1 \\
j
\end{array}\right)^{-1} \\
& \times \sum_{i=0}^{l-j} i !\left(\begin{array}{c}
l-j \\
i
\end{array}\right)\left(\begin{array}{c}
l+m-r-2 \\
i
\end{array}\right) c_{l+m-i-r+1} \\
& -(l+m-r-1) \frac{(-1)^{l+r}}{r}\left(\begin{array}{c}
l \\
r-1
\end{array}\right) \sum_{i=0}^{l-r+1} i !\left(\begin{array}{c}
l-r+1 \\
i
\end{array}\right)\left(\begin{array}{c}
l+m-r-1 \\
i
\end{array}\right) c_{l+m-i-r+1} \\
& +\sum_{j=0}^{r-3} \frac{(-1)^{l+j+1}}{r-1}\left(\begin{array}{l}
l \\
j
\end{array}\right)\left(\begin{array}{c}
m \\
r-j-2
\end{array}\right)\left(\begin{array}{c}
r-2 \\
j
\end{array}\right)^{-1} \\
& \times \sum_{i=0}^{l-j} i !\left(\begin{array}{c}
l-j \\
i
\end{array}\right)\left(\begin{array}{c}
l+m-r-1 \\
i
\end{array}\right) c_{l+m-i-r+2} \\
& +\frac{(-1)^{l+r-1}}{r-1}\left(\begin{array}{c}
l \\
r-2
\end{array}\right) \sum_{i=0}^{l-r+2} i !\left(\begin{array}{c}
l-r+2 \\
i
\end{array}\right)\left(\begin{array}{c}
l+m-r \\
i
\end{array}\right) c_{l+m-i-r+2} \\
& -\sum_{j=0}^{r-2} \frac{(-1)^{l+j+1}}{r}\left(\begin{array}{l}
l \\
j
\end{array}\right)\left(\begin{array}{c}
m+1 \\
r-j-1
\end{array}\right)\left(\begin{array}{c}
r-1 \\
j
\end{array}\right)^{-1}
\end{aligned}
$$


140

T. Komatsu

$$
\begin{aligned}
& \times \sum_{i=0}^{l-j} i !\left(\begin{array}{c}
l-j \\
i
\end{array}\right)\left(\begin{array}{c}
l+m-r-1 \\
i
\end{array}\right) c_{l+m-i-r+2} \\
& -\frac{(-1)^{l+r}}{r}\left(\begin{array}{c}
l \\
r-1
\end{array}\right) \sum_{i=0}^{l-r+1} i !\left(\begin{array}{c}
l-r+1 \\
i
\end{array}\right)\left(\begin{array}{c}
l+m-r \\
i
\end{array}\right) c_{l+m-i-r+2} \\
= & \sum_{j=0}^{r-2} \frac{(-1)^{l+j}}{r}\left(\begin{array}{c}
l+1 \\
j
\end{array}\right)\left(\begin{array}{c}
m \\
r-j-1
\end{array}\right)\left(\begin{array}{c}
r-1 \\
j
\end{array}\right) \\
& \times \sum_{i=0}^{l-j+1} i !\left(\begin{array}{c}
l-j+1 \\
i
\end{array}\right)\left(\begin{array}{c}
l+m-r-1 \\
i
\end{array}\right) c_{l+m-i-r+2} \\
& +\frac{(-1)^{l+r+1}}{r}\left(\begin{array}{c}
l+1 \\
r-1
\end{array}\right) \sum_{i=0}^{l-r+2} i !\left(\begin{array}{c}
l-r+2 \\
i
\end{array}\right)\left(\begin{array}{c}
l+m-r \\
i
\end{array}\right) c_{l+m-i-r+2} \\
= & a_{l+1, m, r} .
\end{aligned}
$$

If $l=3$ in Proposition 5 we have

$$
\begin{aligned}
\hat{c}^{\prime \prime \prime}(x) & \hat{c}^{(m)}(x) \\
= & -\frac{3 ! m ! x}{(m+4) !} \hat{c}^{(m+4)}(x)-\frac{3 ! m !}{(m+3) !} \hat{c}^{(m+3)}(x) \\
& +\sum_{k=0}^{m-4}\left(\begin{array}{c}
m \\
k
\end{array}\right)\left(\frac{c_{k+4}}{k+4}+\frac{3(k+2) c_{k+3}}{k+3}+3(k+1) c_{k+2}+(k+2) k c_{k+1}\right) \\
& \times \frac{x}{(1+x)^{k+4}} \hat{c}^{(m-k)}(x) \\
& -3 \sum_{k=0}^{m-4}\left(\begin{array}{c}
m \\
k
\end{array}\right)\left(\frac{c_{k+3}}{k+3}+2 c_{k+2}+(k+2) c_{k+1}\right) \frac{1}{(1+x)^{k+4}} \hat{c}^{(m-k)}(x) \\
& +\left(\frac{m^{2}-4 m+6}{3 !} c_{m+1}+\frac{(m-1)^{2}(m-2)}{2} c_{m}+\frac{m(m-1)(m-2)^{2}}{2} c_{m-1}\right. \\
& \left.+\frac{m(m-1)^{2}(m-2)(m-3)}{3 !} c_{m-2}\right) \frac{x}{(1+x)^{m+1}} \hat{c}^{\prime \prime \prime}(x) \\
& -\left(\frac{(m-1)(m-2)}{2}\left(c_{m}+2 m c_{m-1}+m(m-1) c_{m-2}-\hat{c}_{m}\right) \frac{1}{(1+x)^{m+1}} \hat{c}^{\prime \prime \prime}(x)\right. \\
& +\frac{1}{2}\left((m-3) c_{m+2}+3 m(m-2) c_{m+1}+3 m(m-1)^{2} c_{m}\right. \\
+ & \left.m m^{2}(m-1)(m-2) c_{m-1}\right) \frac{x}{(1+x)^{m+2}} \hat{c}^{\prime \prime}(x) \\
& -\frac{3}{2}\left((m-2) c_{m+1}+2 m(m-1) c_{m}+m^{2}(m-1) c_{m-1}\right. \\
+ & \left.2\left(\hat{c}_{m+1}+(m+1) \hat{c}_{m}\right)\right) \frac{1}{(1+x)^{m+2}} \hat{c}^{\prime \prime}(x) \\
& \\
& \\
&
\end{aligned}
$$




$$
\begin{aligned}
& \times \frac{x}{(1+x)^{m+3}} \hat{c}^{\prime}(x)-3\left(c_{m+2}+2(m+1) c_{m+1}+(m+1) m c_{m}\right. \\
& \left.-\left(\hat{c}_{m+2}+2(m+2) \hat{c}_{m+1}+(m+2)(m+1) \hat{c}_{m}\right)\right) \frac{1}{(1+x)^{m+3}} \hat{c}^{\prime}(x) \\
& -\left(\hat{c}_{m+3}+3(m+3) \hat{c}_{m+2}+3(m+3)(m+2) \hat{c}_{m+1}+(m+3)(m+2)(m+1) \hat{c}_{m}\right) \\
& \times \frac{\hat{c}(x)}{(1+x)^{m+4}} .
\end{aligned}
$$

By using Proposition 5, we obtain explicit expressions for $\left(\hat{c}_{l}+\hat{c}_{m}\right)^{n}$.

THEOREM 5. Let $l, m, n$ be integers with $m \geq l \geq 1$ and $n \geq 0$. Then

$$
\begin{aligned}
\left(\hat{c}_{l}+\right. & \left.\hat{c}_{m}\right)^{n} \\
= & -\frac{l ! m !}{(l+m+1) !}(n+l+m+1) \hat{c}_{n+l+m} \\
& +\sum_{k=0}^{m-l-1} \sum_{i=0}^{n} \frac{(-1)^{i-1} n !}{(n-i) !}\left(\begin{array}{c}
l+k+i \\
i
\end{array}\right)\left(\frac{i}{l+k+i} a_{l, m, m-k}-b_{l, m, m-k}\right) \hat{c}_{n+m-k-i} \\
& +\sum_{k=0}^{l} \sum_{i=0}^{n} \frac{(-1)^{i-1} n !}{(n-i) !}\left(\begin{array}{c}
m+k+i \\
i
\end{array}\right)\left(\frac{i}{m+k+i} a_{l, m, l-k}-b_{l, m, l-k}\right) \hat{c}_{n+l-k-i} .
\end{aligned}
$$

where $a_{l, m, r}$ and $b_{l, m, r}$ are defined in Proposition 5.

Proof. By (13) and (15) we have

$$
\begin{aligned}
\left(\hat{c}_{l}+\right. & \left.\hat{c}_{m}\right)^{n} \\
= & -\frac{l ! m !}{(l+m+1) !} n \hat{c}_{n+l+m}-\frac{l ! m !}{(l+m) !} \hat{c}_{n+l+m} \\
& +\sum_{k=0}^{m-l-1} a_{l, m, m-k} \sum_{i=0}^{\infty}(-1)^{i}\left(\begin{array}{c}
l+k+i \\
i
\end{array}\right) \frac{n !}{(n-i-1) !} \hat{c}_{n+m-k-i-1} \\
& +\sum_{k=0}^{m-l-1} b_{l, m, m-k} \sum_{i=0}^{\infty}(-1)^{i}\left(\begin{array}{c}
l+k+i \\
i
\end{array}\right) \frac{n !}{(n-i) !} \hat{c}_{n+m-k-i} \\
& +\sum_{k=0}^{l} a_{l, m, l-k} \sum_{i=0}^{\infty}(-1)^{i}\left(\begin{array}{c}
m+k+i \\
i
\end{array}\right) \frac{n !}{(n-i-1) !} \hat{c}_{n+l-k-i-1} \\
& +\sum_{k=0}^{l} b_{l, m, l-k} \sum_{i=0}^{\infty}(-1)^{i}\left(\begin{array}{c}
m+k+i \\
i
\end{array}\right) \frac{n !}{(n-i) !} \hat{c}_{n+l-k-i} \\
= & -\frac{l ! m !}{(l+m+1) !}(n+l+m+1) \hat{c}_{n+l+m} \\
& +\sum_{k=0}^{m-l-1} \sum_{i=0}^{n} \frac{(-1)^{i-1} n !}{(n-i) !}\left(\begin{array}{c}
l+k+i \\
i
\end{array}\right)\left(\frac{i}{l+k+i} a_{l, m, m-k}-b_{l, m, m-k}\right) \hat{c}_{n+m-k-i} \\
& +\sum_{k=0}^{l} \sum_{i=0}^{n} \frac{(-1)^{i-1} n !}{(n-i) !}\left(\begin{array}{c}
m+k+i \\
i
\end{array}\right)\left(\frac{i}{m+k+i} a_{l, m, l-k}-b_{l, m, l-k}\right) \hat{c}_{n+l-k-i} .
\end{aligned}
$$




\section{Remarks}

If $l=1$ in Theorem 5 , we have

$$
\begin{aligned}
\left(\hat{c}_{1}+\hat{c}_{m}\right)^{n}= & -\frac{n+m+2}{(m+2)(m+1)} \hat{c}_{n+m+1} \\
& +\sum_{k=0}^{m-2} \sum_{i=0}^{n-1} \frac{(-1)^{i} n !}{(n-i-1) !}\left(\begin{array}{c}
k+i+1 \\
i
\end{array}\right) a_{1, m, m-k} \hat{c}_{n+m-k-i-1} \\
& -\sum_{k=0}^{m-2} \sum_{i=0}^{n} \frac{(-1)^{i-1} n !}{(n-i) !}\left(\begin{array}{c}
k+i+1 \\
i
\end{array}\right) b_{1, m, m-k} \hat{c}_{n+m-k-i} \\
& +\sum_{i=0}^{n-1} \frac{(-1)^{i} n !}{(n-i-1) !}\left(\begin{array}{c}
m+i \\
i
\end{array}\right) a_{1, m, 1} \hat{c}_{n-i} \\
& +\sum_{i=0}^{n-1} \frac{(-1)^{i} n !}{(n-i-1) !}\left(\begin{array}{c}
m+i+1 \\
i
\end{array}\right) a_{1, m, 0} \hat{c}_{n-i-1} \\
& -\sum_{i=0}^{n} \frac{(-1)^{i-1} n !}{(n-i) !}\left(\begin{array}{c}
m+i \\
i
\end{array}\right) b_{1, m, 1} \hat{c}_{n-i+1} \\
& -\sum_{i=0}^{n} \frac{(-1)^{i-1} n !}{(n-i) !}\left(\begin{array}{c}
m+i+1 \\
i
\end{array}\right) b_{1, m, 0} \hat{c}_{n-i} .
\end{aligned}
$$

Since

$$
\begin{aligned}
a_{1, m, m-k} & =\left(\begin{array}{c}
m \\
k
\end{array}\right)\left(\frac{c_{k+2}}{k+2}+\frac{k c_{k+1}}{k+1}\right), \\
b_{1, m, m-k} & =-\left(\begin{array}{c}
m \\
k
\end{array}\right) \frac{c_{k+1}}{k+1}, \\
a_{1, m, 1} & =c_{m+1}+(m-1) c_{m}, \\
a_{1, m, 0} & =0, \\
b_{1, m, 1} & =\hat{c}_{m}-c_{m}, \\
b_{1, m, 0} & =-\hat{c}_{m+1}-(m+1) \hat{c}_{m},
\end{aligned}
$$

we obtain Theorem 3 .

If $l=2$ in Theorem 5, we have

$$
\begin{aligned}
\left(\hat{c}_{2}+\hat{c}_{m}\right)^{n}= & -\frac{n+m+3}{(m+3)(m+2)(m+1)} \hat{c}_{n+m+2} \\
& +\sum_{k=0}^{m-3} \sum_{i=0}^{n-1} \frac{(-1)^{i} n !}{(n-i-1) !}\left(\begin{array}{c}
k+i+2 \\
i
\end{array}\right) a_{2, m, m-k} \hat{c}_{n+m-k-i-1} \\
& -\sum_{k=0}^{m-2} \sum_{i=0}^{n} \frac{(-1)^{i-1} n !}{(n-i) !}\left(\begin{array}{c}
k+i+2 \\
i
\end{array}\right) b_{2, m, m-k} \hat{c}_{n+m-k-i}
\end{aligned}
$$




$$
\begin{aligned}
& +\sum_{i=0}^{n-1} \frac{(-1)^{i} n !}{(n-i-1) !}\left(\begin{array}{c}
m+i \\
i
\end{array}\right) a_{2, m, 2} \hat{c}_{n-i+1} \\
& +\sum_{i=0}^{n-1} \frac{(-1)^{i} n !}{(n-i-1) !}\left(\begin{array}{c}
m+i+1 \\
i
\end{array}\right) a_{2, m, 1} \hat{c}_{n-i} \\
& +\sum_{i=0}^{n-1} \frac{(-1)^{i} n !}{(n-i-1) !}\left(\begin{array}{c}
m+i+2 \\
i
\end{array}\right) a_{2, m, 0} \hat{c}_{n-i-1} \\
& -\sum_{i=0}^{n} \frac{(-1)^{i-1} n !}{(n-i) !}\left(\begin{array}{c}
m+i \\
i
\end{array}\right) b_{2, m, 2} \hat{c}_{n-i+2} \\
& -\sum_{i=0}^{n} \frac{(-1)^{i-1} n !}{(n-i) !}\left(\begin{array}{c}
m+i+1 \\
i
\end{array}\right) b_{2, m, 1} \hat{c}_{n-i+1} \\
& -\sum_{i=0}^{n} \frac{(-1)^{i-1} n !}{(n-i) !}\left(\begin{array}{c}
m+i+2 \\
i
\end{array}\right) b_{2, m, 0} \hat{c}_{n-i} .
\end{aligned}
$$

Since

$$
\begin{aligned}
a_{2, m, m-k} & =-\left(\begin{array}{c}
m \\
k
\end{array}\right)\left(\frac{c_{k+3}}{k+3}+\frac{2(k+1) c_{k+2}}{k+2}+c_{k+1}\right), \\
b_{2, m, m-k} & =2\left(\begin{array}{c}
m \\
k
\end{array}\right)\left(\frac{c_{k+2}}{k+2}+c_{k+1}\right), \\
a_{2, m, 2} & =\left(-\frac{m}{2}+1\right) c_{m+1}-(m-1)^{2} c_{m}-\frac{m(m-1)(m-2)}{2} c_{m-1}, \\
a_{2, m, 1} & =-c_{m+2}-2 m c_{m+1}-m(m-1) c_{m}, \\
a_{2, m, 0} & =0 \\
b_{2, m, 2} & =(m-1) c_{m}+m(m-1) c_{m-1}+\hat{c}_{m}, \\
b_{2, m, 1} & =2 c_{m+1}+2 m c_{m}-2 \hat{c}_{m+1}-2(m+1) \hat{c}_{m}, \\
b_{2, m, 0} & =\hat{c}_{m+2}+2(m+2) \hat{c}_{m+1}+(m+2)(m+1) \hat{c}_{m},
\end{aligned}
$$

we obtain Theorem 4 .

\section{REFERENCES}

[1] T. Agoh and K. Dilcher. Convolution identities and lacunary recurrences for Bernoulli numbers. J. Number Theory 124 (2007), 105-122.

[2] T. Agoh and K. Dilcher. Higher-order recurrences for Bernoulli numbers. J. Number Theory 129 (2009), 1837-1847.

[3] T. Agoh and K. Dilcher. Recurrence relations for Nörlund numbers and Bernoulli numbers of the second kind. Fibonacci Quart. 48 (2010), 4-12.

[4] L. Comtet. Advanced Combinatorics. Reidel, Dordrecht, 1974.

[5] K. Dilcher. Sums of products of Bernoulli numbers. J. Number Theory 60 (1996), 23-41.

[6] I. M. Gessel. On Miki's identity for Bernoulli numbers. J. Number Theory 110 (2005), 75-82.

[7] R. L. Graham, D. E. Knuth and O. Patashnik. Concrete Mathematics, 2nd edn. Addison-Wesley, Reading, MA, 1994.

[8] T. Komatsu. Poly-Cauchy numbers. Kyushu J. Math. 67 (2013), 143-153. 
[9] T. Komatsu. Convolution identities for Cauchy numbers. Acta Math. Hungar. 144 (2014), 76-91.

[10] D. Merlini, R. Sprugnoli and M. C. Verri. The Cauchy numbers. Discrete Math. 306 (2006), 1906-1920.

[11] S. Roman. The Umbral Calculus. Dover, New York, 2005.

[12] F.-Z. Zhao. Sums of products of Cauchy numbers. Discrete Math. 309 (2009), 3830-3842.

\author{
Takao Komatsu \\ School of Mathematics and Statistics \\ Wuhan University \\ Wuhan, 430072 \\ China \\ (E-mail:komatsu@whu.edu.cn)
}

